\title{
Il Sommo Topolino nella selva oscura. Spunti per una lettura linguistica de L'Inferno di Topolino
}

\author{
Daniela Pietrini \\ Martin Luther University Halle-Wittenberg \\ daniela.pietrini@romanistik.uni-halle.de
}

\section{Riassunto}

La Divina Commedia non ha soltanto condizionato fin dalle origini l'intero percorso della letteratura e della lingua italiana, ma continua ancora oggi a ispirare opere e autori dagli stili più diversi, dalla musica al cinema e ovviamente al fumetto. All'interno della vasta produzione di fumetti ispirati alla Commedia dantesca, questo contributo si concentra sul fumetto Disney "L'Inferno di Topolino", parodia dell' Inferno dantesco realizzata da Guido Martina (testi) e Angelo Bioletto (disegni) per i numeri 7-I2 di Topolino (I949-I950) nonché capostipite del fortunato filone delle cosiddette "Grandi Parodie Disney". L'obiettivo è proporre una lettura linguistica della parodia disneyana: dopo aver brevemente elencato i principali tratti distintivi del genere parodia nel suo insieme, si procede all'analisi de "L'Inferno di Topolino" focalizzando l'attenzione da un lato sulle modalità di realizzazione linguistica dell'intento parodico e dall'altro sullo specifico fumettistico della trasposizione disneyana. Si mettono così in evidenza diverse strategie di riproduzione / imitazione dell'italiano antico dal punto di vista morfologico, sintattico e lessicale.

Parole chiave: parodia; Disney; fumetto; Inferno; italiano antico; rapporto testo e immagine.

\begin{abstract}
The Divine Comedy has not only had a profound influence on Italian Literature and Language since the very beginning, but it also continues to be a source of inspiration for many authors and works of different styles and cultural fields, from music to film etc. Within the vast amount of comics inspired by Dante's masterpiece, this paper focuses on the parody of Dante's Inferno "L'Inferno di Topolino" ("Mickey's Inferno"). This comic book story was created in Italy back in 1949-50 for the Topolino Magazine by Guido Martina (writer) and Angelo Bioletto (cartoonist), two of the first comic book artists of Disney Italian comics, and it is the very first of the famous Disney Great Parodies. This study proposes an interpretation of this Disney parody from a linguistic perspective. After listing the main distinguishing features of parody as a whole, it provides an analysis of the comic book history "L'inferno di Topolino" by focusing on the linguistic techniques used to accomplish the parodic purpose on the one hand, and on the specific of a comic book parody on the other. Many strategies of imitating old Italian morphology, syntax and lexicon are pointed out.
\end{abstract}

Key words: parody, Disney; comic; Hell; old Italian; text and image relationship. 


\section{INTRODUZIONE}

T a Divina Commedia non ha soltanto condizionato fin dalle origini l'intero L percorso della letteratura e della lingua italiana, ma continua ancora oggi a ispirare opere e autori dagli stili più diversi, dalla musica heavy metal, hiphop o pop (per restare in Italia, si pensi all'album del 2007 Pia come la canto io della cantautrice Gianna Nannini dedicato al personaggio del Purgatorio dantesco Pia de' Tolomei) al cinema hollywoodiano (ad esempio le vicende del serial killer protagonista di Seven di David Fincher o l'interpretazione del suicidio di Pier delle Vigne riproposta nel thriller Hannibal di Ridley Scott). ${ }^{\mathrm{I}}$ In questo proliferare di opere ispirate più o meno liberamente alla Commedia dantesca spiccano anche numerosi fumetti. ${ }^{2}$

Tra questi, un posto di tutto rilievo spetta a "L'Inferno di Topolino", reinterpretazione disneyana della prima cantica della Divina Commedia frutto della collaborazione tra Guido Martina (testi) e Angelo Bioletto (disegni). Questo fumetto, pubblicato per la prima volta a puntate sui numeri 7-I2 di Topolino tra l'autunno I949 e la primavera I950, costituisce non solo la prima storia made in Italy creata appositamente per il Topolino nel suo nuovo formato tascabile, ${ }^{3}$ ma anche il capostipite del fortunato filone delle cosiddette "Grandi Parodie Disney", rivisitazioni in chiave paperesca e topesca dei classici della letteratura nonché, in un secondo momento, di opere cinematografiche e serie televisive. ${ }^{4}$

I. Si sono moltiplicati, soprattutto in corrispondenza del $750^{\circ}$ anniversario della nascita di Dante, i saggi in versione cartacea o digitale dedicati ai rapporti tra il poeta fiorentino e la cultura pop (si leggano a titolo di esempio almeno Chiamenti (s.d.); Cotugno, A./Gargano, T. (20I6); Garavelli (s.d.) e diversi articoli di giornale come Buttitta 20I5). Non è certo un caso che il noto dantista Marco Santagata in svariate occasioni abbia evidenziato il ruolo di Dante nel circuito della comunicazione contemporanea definendolo una sorta di "icona pop" (cfr. Napolitano 2016).

2. Si citino almeno il recente "La porta dell'Inferno" (Speciale Dampyr, I2, 20I6,) e ancora, sempre per gli amanti del giallo-misterioso-fantasy, "Discesa all'Inferno" (Lazarus Ledd, 89, 2000,) e - per gli anni Novanta - "Inferno" (Nathan Never, Io) e "Diavoli dell'Inferno" (Martin Mystère, I53). Cambiando genere e target, ricordiamo ancora le avventure del diavolo buono Geppo in "Inferno 2000", fumetto uscito per la prima volta nel 1984 e riproposto qualche anno fa dallo stesso autore in una versione ripulita a tiratura limitata (solo 200 copie, Dossi 20II), le tre avventure di Cattivik ambientate rispettivamente all'Inferno ("Un'avventura infernale", Cattivik, 32, 1992), in Purgatorio ("Cattivik in Purgatorio", Cattivik, 40, 1993) e in Paradiso ("Cattivik in Paradiso", Cattivik, 54, 1994), la dissacrante Rovina in commedia di Jacovitti del lontano 1947 o ancora la celeberrima Commedia a fumetti di Toninelli, anch'essa recentemente ripubblicata in volume unico. La panoramica appena schizzata si limita consapevolmente alla cultura italiana, per quanto non manchino nemmeno nelle altre lingue, non solo europee, pregevoli rivisitazioni a fumetti del poema dantesco sotto forma di manga, graphic novel ecc.

3. Nell'aprile 1949 il Topolino cambia formato e periodicità passando da settimanale a mensile e trasformandosi nel primo tascabile dell'editoria a fumetti. Cfr. Becattini/Boschi/Gori/Sani 2OI2: 46-50.

4. Il filone parodico dei Disney made in Italy inaugurato da Martina e Bioletto nel 1949 ha goduto di fortuna enorme e non è mai stato abbandonato dagli autori italiani, per quanto 
All'interno della vasta produzione di fumetti ispirati alla Commedia dantesca, questo contributo propone una lettura linguistica della parodia disneyana: dopo aver brevemente elencato i principali tratti distintivi del genere parodia nel suo insieme, si procederà all'analisi de "L'Inferno di Topolino" focalizzando l'attenzione da un lato sulle modalità di realizzazione linguistica dell'intento parodico e dall'altro sullo specifico fumettistico della trasposizione disneyana.

\section{Fumetto E PAROdia}

Fumetto e parodia sono due generi che vanno spesso di pari passo, anzi la vena parodica sembra attraversare l'intera storia del fumetto - almeno per quanto riguarda quello europeo - fin dalle sue origini. Seguendo il percorso tracciato da Thierry Groensteen (2010) in occasione della mostra "Parodies, la bande dessinée au second degré" organizzata a Angoulême dalla Cité internationale de la bande dessinée et de l'image ( 5 gennaio - 24 aprile 20II) e dedicata al rapporto costitutivo tra fumetto e parodia, già le primissime storie dello svizzero Rudolphe Töpffer, considerato ormai dai più il padre del fumetto, possono essere lette come parodie di alcuni generi letterari alla moda, così come i disegni di questi protofumetti sembrano parodizzare le pose melodrammatiche degli attori degli anni Trenta dell'Ottocento:

Quand Rodolphe Töpffer publie ses premières 'histoires en estampes' dans les années 1830, non seulement ses récits parodient certains genres littéraires en vogue (le roman pastoral, le roman picaresque, le voyage d'instruction), mais ses dessins parodient aussi les poses mélodramatiques des acteurs de l'époque. Il semble bien qu'aux origines, le personnage de bande dessinée comique a été une parodie d'acteur!" (Groensteen 20IO).

Molto meno trasparente è invece la nozione stessa di parodia, caratterizzata da un forte divario tra il senso tecnico del termine e il suo significato corrente. Nell'uso comune il termine parodia ha un'accezione molto generica indicando qualsiasi "versione comica, caricaturale di un'opera letteraria, di una canzone, di un film" (Sabatini/Coletti 2005, s.v.) o addirittura, per estensione, l'“imitazione caricaturale di qualcuno o qualcosa" (ibid.). Nell'italiano colloquiale parodia assume quindi il valore di una sorta di epiteto passe-partout equivalente a comico o umoristico. Nell'uso popolare il termine tende addirittura verso una connotazione dispregiativa o comunque restrittiva nel senso di un'imitazione grossolana e di scarso valore, spesso degradante, la cui finalità

i risultati raggiunti siano qualitativamente alterni. Sulle modalità dell'innesto parodico e sugli autori principali delle parodie Disney cfr. Becattini/Boschi/Gori/Sani 20I2: 393-396 e Frezza 1987 e 1989: I256-I259. Un elenco cronologico delle parodie Disney pubblicate finora è disponibile sul sito PaperPedia Wiki alla voce "Parodie Disney"(http://it.paperpedia.wikia. com/wiki/Parodie_Disney). 
consisterebbe solo nell'abbassamento del livello del testo di riferimento. 5 L'incertezza semantica e definitoria caratterizza la parodia anche nel linguaggio colto, che spesso la confonde con il pastiche o la satira e la condanna a vario titolo: in quanto discorso parassita a spese degli autori originali, come discorso menzognero e ingannevole, in quanto pratica degradante volta a svalutare i propri bersagli oppure, nella migliore delle ipotesi, come genere insulso di mero svago (cfr. Sangsue 2006: I5-16). Non potendo entrare nel merito delle diverse teorie della parodia (Bachtin, Rose, Hutcheon, Genette ecc., per la cui trattazione si rimanda proprio al saggio di Sangsue 2006), ci limitiamo a sottolineare, nel persistere di una tradizione che lega la parodia al comico, come nell'antichità la parodia fosse non solo un genere letterario, ma anche una tecnica di citazione limitata alla ricontestualizzazione di un frammento testuale senza necessità di intervenire sulla lettera del testo originario. ${ }^{6}$

Nell'ambito di questo articolo intendiamo per parodia una pratica ipertestuale nel senso indicato da Genette (1982/1997, 7-8) di relazione tra un ipertesto B (il testo parodico, nel nostro caso il fumetto disneyano) e un ipotesto preesistente A (la Commedia dantesca), 7 sul quale il primo si innesta trasformandolo però non solo in funzione ludica - come voleva Genette -, ma anche comica o satirica (secondo la visione meno restrittiva di Sangsue 2006: 78). Il gesto parodico così definito, ponendosi tra la dissacrazione dell'ipotesto e l'ammirazione verso di esso, è costitutivamente ambiguo in quanto il rovesciamento di un testo preesistente ne implica comunque il riconoscimento: "la parodia funzionerebbe dunque come una sorta di ammirazione obliqua, un omaggio che non vuole dichiararsi tale." (Sangsue 2006: 80) Proprio questo aspetto di consacrazione implicita nella smitizzazione spiega la preferenza per i grandi capolavori letterari e artistici come oggetto della trasformazione parodica. Non è quindi un caso che la parodia disneyana scelga proprio la Divina Commedia come primo ipotesto di una lunga serie di fortunate parodie. ${ }^{8}$

5. Non a caso il Sabatini/Coletti (2005, s.v.) riporta anche il significato figurato di "riproduzione scadente e ridicola di ciò che una cosa, una persona o un'istituzione dovrebbe essere in realtà" indicandone come sinonimi i termini burla e farsa. Anche il francese lascia registrare connotazioni e significati analoghi: come osserva giustamente proprio Sangsue (2006: I4), nel discorso letterario e scolastico francese la parodia ha il significato dispregiativo e restrittivo di caricatura (“imitation grossière qui ne restitue que certaines apparences", TLFi, s.v.).

6. La ricontestualizzazione bastava a garantire l'effetto ricercato (cfr. Householder 1944). Si veda anche Quintiliano: "seu ficti notis versibus similes, quae $\pi \alpha \rho \omega \delta i ́ \alpha$ dicitur" ("versi simili a quelli autentici: questa è la parodia", Quintilianus, De Institutione oratoria VI, 3, 97).

7. Oltre alla definizione restrittiva di intertestualità di Genette come „presenza effettiva di un testo in un altro" (Id., 4), cfr. anche Kristeva 1978 (II9-I43). Genette distingue due modalità fondamentali di relazione ipertestuale (la trasformazione di un testo e l'imitazione di uno stile) e tre regimi (ludico, satirico e serio) costruendo così uno schema a sei caselle delle pratiche ipertestuali in cui la parodia occupa la casella della trasformazione testuale in funzione ludica.

8. Per restare nell'ambito dell'Inferno dantesco citiamo ancora la storia Paolino Pocatesta e la bella Franceschina, parodia dell'episodio di Paolo e Francesca del V canto dell'Inferno 


\section{La Divina Parodia: dall'ipotesto all'ipertesto}

La possibilità di esprimere ammirazione verso un modello prendendone tuttavia le distanze in maniera anche dissacrante non è l'unica ragione che fa delle grandi opere della letteratura un archetipo particolarmente stimolante per l'operazione parodica. La parodia interviene di preferenza su capolavori di grande fama anche perché proprio la loro notorietà contribuisce a garantire la riuscita dello stesso stravolgimento parodico. Perché una parodia funzioni è infatti necessario che il lettore sia in grado di smascherare il gioco del travestimento, ossia di riconoscere l'ipotesto che si cela sotto l'ipertesto. Anzi, il mero riconoscimento dell'ipotesto non basta: è indispensabile che il lettore abbia una conoscenza relativamente approfondita dell'ipotesto in questione per cogliere lo scarto che lo separa dal suo ipertesto parodico individuando differenze, deviazioni, anacronismi, deformazioni ecc. ${ }^{9}$

Quanto all'operazione disneyana di mascheramento parodico della Divina Commedia, sono molteplici le ragioni che si celano dietro la scelta dell'ipotesto dantesco. Non va sottovalutato innanzitutto lo sfondo vagamente pedagogico dei primi fumetti italiani, tenuto conto anche del fatto che in Italia, a differenza di quanto avviene ad esempio negli USA, il fumetto nasce programmaticamente come genere della letteratura per l'infanzia, pubblicato inizialmente non sulle pagine dei quotidiani, ma su un periodico apposito dal titolo emblematico di Corriere dei Piccoli (cfr. almeno Pietrini 2009, 26-33, e la bibliografia ivi citata). Ancora fino agli anni Quaranta il fumetto è visto da molti come un genere inferiore della letteratura per l'infanzia, spesso stigmatizzato, sminuito se non addirittura avversato (cfr. Detti 1984). La scelta, nei primi anni di produzione delle storie Disney nostrane, di un archetipo come il poema dantesco si spiega solo in parte alla luce della considerazione del ruolo della Divina Commedia quale testo base dell'istruzione scolastica italiana, in quanto presumibilmente noto ai potenziali lettori del fumetto (allievi delle scuole), a garanzia della riuscita del travestimento parodico e della relativa decodifica. Ma la scelta dell'ipotesto dantesco, proprio per il duplice valore del gesto parodico di smitizzazione e omaggio insieme, risponde probabilmente anche al tentativo di nobilitare il fumetto stesso, genere considerato inferiore, mettendolo in relazione - sia pur parodica - con un capolavoro unanimemente riconosciuto quale la cantica di Dante.

pubblicata su Topolino nel I980, e una seconda parodia dell'intero Inferno che vede stavolta protagonista Paperino (L'Inferno di Paperino del 1987), oltre a un'illustrazione di Marco Rota intitolata Paperante Alighieri che rappresenta Paperino nelle vesti di Dante con in braccio la sua "Anatrina Commedia", pubblicata per la prima volta nel 1986 su Paperino mese 70 (cfr. inducks.org).

9. Come sottolinea giustamente Geneviève Idt, durante la decodifica di un testo parodico il lettore compie tre azioni successive: I) riconosce la presenza di un altro testo nel testo; 2) identifica questo ipotesto; 3) misura lo scarto tra ipotesto e ipertesto (Idt 1972-73: I48). 


\section{1. Gli indizi paratestuali, iconici e testuali della parodia disneyana}

Se la riuscita dell'operazione parodica dipende in gran parte dalle capacità ermeneutiche di decodifica del destinatario della parodia, dal canto proprio l'autore cerca di indirizzare il lettore al riconoscimento dell'intento parodico e all'identificazione dell'ipotesto prescelto. Nel caso specifico de "L'Inferno di Topolino" gli autori non si limitano a disseminare l'incipit della storia di indizi di tipo paratestuale e testuale che guidino il lettore alla decodifica della parodia e del suo ipotesto. Trattandosi di un fumetto, genere il cui linguaggio è basato sull'interazione di un elemento verbale con un disegno, un ruolo di primo piano spetta agli indizi "disegnati", cioè di tipo iconico.

Il primo degli indizi paratestuali è il titolo. In "L'Inferno di Topolino" (come anche, 38 anni dopo, in "L'Inferno di Paperino") il titolo originario dell'ipotesto, cioè il nome della cantica dantesca "Inferno", è mantenuto, ma con l'aggiunta di un sintagma preposizionale in funzione di modificatore postnominale (rispettivamente "di Topolino" o "di Paperino") che attribuisce questa particolare versione dell' Inferno al personaggio disneyano volta per volta protagonista del rovesciamento ipertestuale. L'indizio fornito al lettore è sì orientativo, ma ancora implicito in quanto il sintagma l'inferno di $X$ potrebbe anche essere letto con il significato figurato, tipico del linguaggio familiare, di situazione di "grave turbamento fisico o morale" o anche di "gran chiasso e confusione" (TREC, s.v.) (si pensi anche alla locuzione aggettivale d'inferno nel senso di "tormentato, agitato, insopportabile" in espressioni come "giornata d'inferno"). Nello specifico fumettistico delle parodie disneyane è fondamentale, per disambiguare il riferimento, l'apporto del disegno, che è nel fumetto un elemento imprescindibile dal testo e che contribuisce a pieno titolo alla narrazione proprio al pari dell'elemento verbale. Nel caso de "L'Inferno di Topolino" il richiamo all'archetipo, implicito nel titolo, diventa evidente nell'iconografia del personaggio di Topolino-Dante. Nel fumetto seriale (come appunto le storie del Topolino) la riconoscibilità esteriore dei personaggi primari, fedeli a sé stessi pur essendo immersi in avventure sempre nuove, è garantita dalla costanza di fisionomia e abbigliamento. Anche la parodia disneyana della cantica dantesca non intacca i caratteri iconici peculiari di Topolino, immediatamente identificabile grazie a orecchie, muso, scarpe e guanti gialli, ma la sua rappresentazione segue la tipizzazione visuale della figurazione fisica di Dante così come essa è presente nell'immaginario collettivo (v. fig. I). Si tratta di un vero e proprio "cliché iconico", ispirato alla descrizione dell'aspetto di Dante tramandataci da Boccaccio (Trattatello e Vita di Dante) e dai ritratti di Giotto e Domenico di Michelino:

Torniamo alla figura di Dante, inconfondibile a partire dall'abbigliamento e dai lineamenti somatici, come il volto e la sua espressione, divenuti cliché 
iconici: l'abito di panno rosso, il lucco, che lo copre interamente avvolgendogli il capo a mo' di moderno cappuccio, sormontato dall'immancabile corona di alloro che gli cinge le tempie e lascia in evidenza il volto, quasi sempre ritratto di profilo [...]. (Pacifico 2015, s.p.)

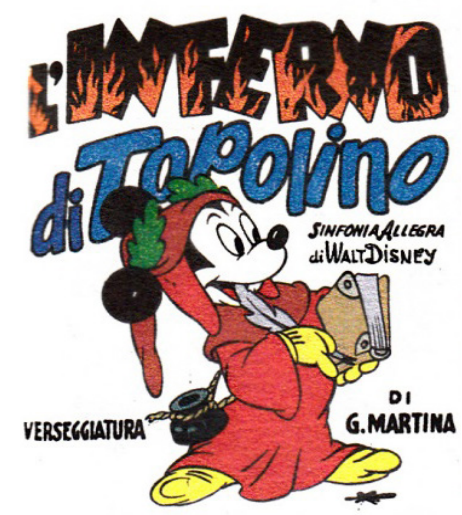

Fig. I. Topolino-Dante, riconoscibile in quanto Topolino grazie a orecchie, muso, scarpe e guanti gialli, e in quanto Dante - tradizionalmente rappresentato di profilo - grazie a lucco ${ }^{\mathrm{IO}}$ rosso, corona d'alloro e attributi da poeta (penna d'oca, librone, calamaio).

Parallelamente gli autori disseminano in apertura de "L'Inferno di Topolino" alcuni indizi testuali del travestimento parodico che però, nello specifico fumettistico, si realizzano a più livelli di testo e nell'interazione con l'elemento disegnato. Il genere fumetto riserva all'elemento verbale diversi spazi: quello più propriamente narrativo della didascalia, solitamente utilizzata dalla voce fuori campo dell'io narrante per fornire informazioni sullo spazio-tempo della vicenda, e le nuvolette di parola, che realizzano la finzione del dialogo facciaa-faccia tra i personaggi uscendo convenzionalmente dalle loro bocche, oltre a eventuali esempi di "testo disegnato" nel senso di lettere, avvisi, insegne, articoli di giornale ecc. integrati nei disegni stessi, e agli innumerevoli ideofoni (cfr. Pietrini 20I4: 86-88). Pertanto gli autori de "L'Inferno di Topolino", nel pieno rispetto dello specifico di una parodia a fumetti, sparpagliano gli indizi necessari alla decodifica della parodia non solo nel titolo (indizio paratestuale) e nell'iconografia del Topolino-Dante del frontespizio (indizio disegnato), ma anche a livello testuale collocandoli in entrambi i luoghi principali della parola nel fumetto, vale a dire didascalia e nuvolette (fig. 2). All'ipotesto dantesco gli autori alludono inserendo nella didascalia immediatamente accanto al

Io. Il lucco è una "veste maschile a pieghe, lunga e accollata, in uso a Firenze nel XIV sec., di panno o damasco spec. rosso o nero, dapprima riservata a nobili, medici, magistrati e sim., poi indossata da tutti i cittadini al di sopra dei 18 anni." (NDMo, s.v.) 
titolo il riferimento al poema di Dante attraverso la sillessi ${ }^{\mathrm{II}}$ "[...] Topolino e Pippo mietevano applausi recitando una grande commedia, anzi una...divina commedia" (sillessi peraltro segnalata anche tipograficamente dal grassetto) e svelando esplicitamente la relazione tra i personaggi dell'ipo- e dell'ipertesto ("Topolino faceva la parte di Dante" e "Pippo faceva Virgilio"). Perché il riconoscimento dell'ipotesto venga assicurato anche a livello dialogico del testo in nuvoletta, l'ipertesto parodico inizia con una citazione della cantica dantesca, pronunciata da Topolino-Dante: "E quindi uscimmo a riveder le stelle!". La scelta di questo verso è tutt'altro che casuale: si tratta dell'ultimo verso dell'Inferno, quindi di una citazione emblematica (l'incipit e la conclusione dei grandi capolavori sono sempre più facilmente memorizzabili e memorizzati) scelta come prima battuta della parodia disneyana per lanciare al lettore dell' $\mathrm{i}$ pertesto un ulteriore segnale a alta riconoscibilità (v. anche $\$ 5$ ).

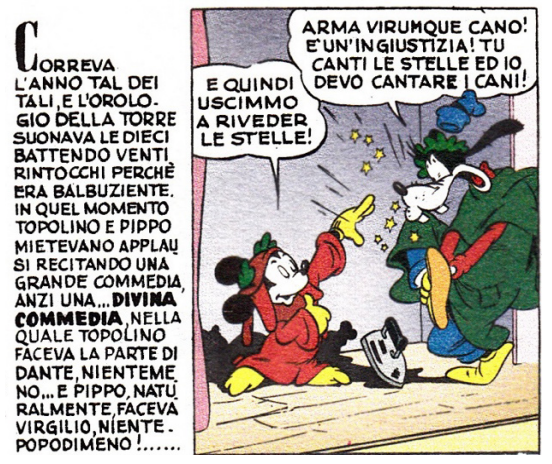

Fig. 2. Gli indizi testuali a guida del lettore nella decodifica del gesto parodico sono inseriti tanto nello spazio propriamente narrativo della didascalia che in quello dialogico delle nuvolette.

Nello stesso tempo gli autori guidano il lettore non solo a identificare l'ipotesto, $\mathrm{ma}$ anche a riconoscere correttamente l'intenzione parodica dell'ipertesto $\left(\mathrm{cfr} . \S_{3}\right)$, cioè a rendersi immediatamente conto di trovarsi di fronte non solo alla trasformazione di un testo preesistente ben preciso (che il lettore accorto avrà appena individuato correttamente nel poema dantesco), ma a un'operazione di rovesciamento con finalità ludica secondo la definizione di cui al $\$ 2$. Ecco quindi, a sottolineare il carattere eminentemente comico della relazione ipertestuale fin dalla prima didascalia, la presenza di una battuta umoristica (l'orologio batte venti rintocchi pur essendo le dieci perché è balbuziente) e di un climax familiare-scherzoso "nientemeno - nientepopodimeno", che ci consente tra l'altro di retrodatare l'avverbio nientepopodimeno almeno al 1949,

II. La sillessi è una figura retorica secondo la quale un termine è usato contemporaneamente in senso proprio e figurato, per es. l'aggettivo carico in "un guerriero carico d'armi e di gloria" (v. TREC, s.v.). 
mentre sia De Mauro che Sabatini/Coletti lo datano 1958. Un analogo meccanismo di climax scherzoso è presente anche a livello dialogico nelle prime nuvolette: alla citazione dell'ultimo verso dantesco da parte di Topolino-Dante segue infatti quella dell'incipit dell'Eneide ("arma virumque cano") ad opera di Pippo-Virgilio. L'intenzione comica è segnalata dal fraintendimento del latino cano (= "canto") confuso con "cane" sulla base di un gioco di parole fondato sulla deformazione mistilingue, per cui Pippo si lamenta di dover cantare i cani invece delle stelle, con evidente abbassamento di tono a scopo ludico.

Ma il gioco di trasformazione e parallelo smascheramento realizzato attraverso il fumetto è molto più complesso e stratificato potendo contare sempre anche sul doppio livello costituito dal richiamo reciproco di testo e immagine. Già nella prima vignetta della prima storia disneyana made in Italy, nonché capostipite della serie delle "Grandi Parodie Disney", gli autori sfruttano a piene mani il potenziale comico della divaricazione tra lettera e disegno giocando con il contrasto tra senso letterale e traslato di locuzioni e termini. Osserviamo bene proprio questa vignetta introduttiva alla trasposizione parodica dell' $I n-$ ferno dantesco: sul palcoscenico Topolino-Dante declama il celeberrimo verso finale della cantica "[...] a riveder le stelle" e Pippo-Virgilio saltella tenendosi in mano un piede dal quale effettivamente escono delle stelline, mentre a terra cade chissà da dove un ferro da stiro! È evidente il gioco tra il senso figurato del testo (in cui il verso dantesco viene smitizzato mettendolo in relazione con la locuzione familiare vedere le stelle nel senso di "provare un acutissimo dolore fisico, per lo più improvviso e temporaneo") e la sua interpretazione letterale a livello di disegno, con la rappresentazione grafica sotto forma di stelline di "quella specie di sfarfallio luminoso davanti agli occhi che si verifica spesso quando si è colpiti da un dolore acuto e improvviso" (Quartu/Rossi 20I2, s.v. "stella").

Il connubio tra fumetto e parodia non solo moltiplica come in un caleidoscopio piani e livelli su cui distribuire gli indizi di decodifica della relazione tra ipotesto e ipertesto (titolo, frontespizio, didascalie narrative e nuvolette dialogiche, livello iconico), ma spinge gli autori a gettare continui ponti tra le singole parti (citazione colta e relativo abbassamento, senso letterale e traslato di versi e modi di dire, deformazione mistilingue), il tutto nell'estrema densità dello spazio circoscritto di titolo e frontespizio (fig. I), prima didascalia e vignetta iniziale (fig. 2).

Le vignette successive valgono a fissare il travestimento dei personaggi seriali (Topolino e Pippo) e a consolidare le modalità dello scarto comico fondato sul divario tra testo e disegno. Tradizione letteraria e conoscenzereminiscenze scolastiche si intrecciano in un accostamento fumetto-Dante- 
scuola che costituisce una delle peculiarità de "L'inferno di Topolino". ${ }^{22}$ In seguito all'incantesimo di un ipnotizzatore presente in sala, Topolino e Pippo rimangono legati ai personaggi appena interpretati. Pippo-Virgilio recita in latino, ma si tratta semplicemente di esercizi di declinazione o di coniugazione elementare (ego sum, rosa-rosae) resi espliciti dall'ammiccamento metalinguistico "foemina-foeminae della prima declinazione", in cui si strizza l'occhio al lettore (presumibilmente non a digiuno di conoscenze di latino di base) sottolineando ancora una volta l'intenzione ludico-parodica (fig. 3 ).
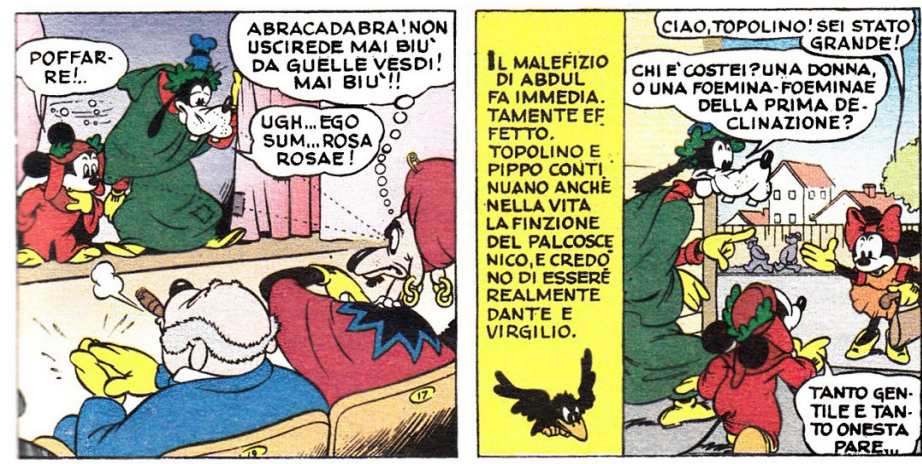

Fig. 3. Le vignette immediatamente successive fissano gli estremi del patto parodico con il lettore legando i personaggi del fumetto seriale al loro travestimento parodico.

La fissazione di Topolino nei panni di Dante avviene invece attraverso la ripetuta citazione di celebri versi danteschi come l'inizio del sonetto della Vita Nova "Tanto gentile e tanto onesta pare", anch'esso materia scolastica obbligatoria e quindi immediatamente riconoscibile per il lettore (presumibilmente ragazzo in età scolare), o più avanti la battuta "o Beatrice ancor non è finita quella commedia ch'era incominciata nel mezzo del cammin di nostra vita!" (v. anche $\$ 5$ ). Resta costante anche lo scarto parodico generato dalla frizione tra testo dialogico e disegno, per cui Minni, che agli occhi del lettore ormai avvezzo al travestimento parodico non può che apparire come una novella Beatrice, si rivela tutt'altro che "gentile", pronta a colpire il malcapitato poeta stilnovista Topolino con ben più prosastici ombrello e borsetta.

I2. Si tenga presente che i prodromi della parodia disneyana dell' Inferno dantesco sono stati individuati proprio in un diario scolastico, il "Diario degli amici di Topolino" del 1948 (n. I3I della collana Gli Albi d'oro), contenente un indovinello demenziale su Dante e un disegno che richiama in maniera evidente una vignetta della successiva parodia (cfr. Brambilla 20I3). Numerosi i riferimenti all'istituzione scolastica anche nell'ambito della parodia stessa, ad es. gli scolari e le pagelle del canto IV, il "serpente scolasticus", i numerosi maestri puniti nell'Inferno ecc. Sul rapporto tra la parodia disneyana dell'Inferno e l'istituzione scolastica v. anche $\$ 3$. 


\section{2. La struttura de "L'Inferno di Topolino"}

La parodia dell'Inferno con Topolino protagonista è strutturata in canti, proprio come l'opera dantesca. I canti sono segnalati esplicitamente da una scritta fuori nuvoletta (una sorta di titolo inserito nella vignetta) fino al canto XXVII, mentre i successivi, non più segnalati, possono essere agevolmente ricostruiti dal lettore sulla base dei personaggi incontrati da Topolino-Dante e Pippo-Virgilio. Mancano alcuni canti come il XIV (quello dei violenti contro Dio) e altri sono uniti insieme, ma nel complesso è possibile seguire con un certo ordine e una relativa completezza il parallelismo con la cantica dantesca. ${ }^{13}$ Il passaggio dalla cornice (la vicenda di Topolino e Pippo attori che, a causa del trucco dell'ipnotizzatore, non possono più svestire i panni rispettivamente di Dante e Virgilio) al travestimento parodico dell'ipotesto dantesco avviene attraverso l'espediente del sonno: i personaggi si addormentano. La parodia è tale solo se mantiene un continuo riferimento all'ipotesto, ed infatti anche nella Divina Commedia Dante non ricorda bene come ha fatto a finire nella selva oscura perché era preso dal sonno: "Io non so ben ridir com 'i" v'intrai, / tant'era pien di sonno a quel punto / che la verace via abbandonai." (If. I, IO-I2). Questo momento viene ripreso in entrambe le parodie disneyane: in "L'Inferno di Topolino" la successione delle battute in nuvoletta crea una sorta di gioco di parole a struttura chiastica tra il significato figurato di morire nel modo di dire "morire di sonno" e il senso proprio del verbo in "sognare di morire", gioco di parole che porta i personaggi effettivamente nel regno dei morti, cioè all'inferno ("Io mi sento morire dal sonno... ahhh!" / "e io... sogno di morire... ohhhh..."), mentre nella parodia di Paperino il personaggio si risveglia e non sa più orientarsi (avendo per così dire "smarrito la diritta via").

Scopo di questo lavoro è illustrare il contributo dell'elemento linguistico alla costruzione e al successo della parodia disneyana dell'Inferno. Partendo dalla premessa che una parodia non può limitarsi a deformare a scopo ludico i contenuti del suo ipotesto, ma deve investirne anche la lettera, nelle pagine che seguono si tralasceranno tutti i sia pur ricchi riferimenti ai personaggi e ai momenti della cantica dantesca per concentrarsi esclusivamente sull'elemento verbale, considerato però nella sua essenza fumettistica, cioè in relazione con il linguaggio iconico dell'elemento disegnato.

13. Manca in questa sede lo spazio per un confronto, pur promettente e di sicuro interesse, con le modalità di realizzazione dell'altra parodia disneyana dell'Inferno dantesco, il più volte citato "Inferno di Paperino". Ci limitiamo quindi a osservare come in quest'ultimo manchi invece la divisione in canti e il tipo di peccatori punito nell'inferno vari considerevolmente rispetto all'originale. Ne "L'Inferno di Paperino" ritroviamo piuttosto i protagonisti di quell"'inferno della civiltà" di cui Paperino stesso parla ai nipotini nel lungo prologo della storia: gli inquinatori, i burocrati, i piromani, coloro che hanno abusato dell'automobile ecc. 


\section{Aspetti linguistici de "L'Inferno di Topolino"}

La parodia degli sceneggiatori disneyani gioca a livello linguistico principalmente con due aspetti: la riproduzione deformante dell'italiano medioevale, $o$ meglio dell'immagine dell'italiano medioevale che gli autori ritenevano condivisa e riconoscibile per il pubblico destinatario del travestimento parodico, e l'imitazione derisoria degli stilemi della poesia dantesca. Le dimensioni ridotte di questo contributo non consentono un'analisi sistematica del testo parodico né un raffronto puntuale tra ipertesto (parodia) e ipotesto (poema). Ci limiteremo pertanto a illustrare alcuni tratti linguistici scelti particolarmente significativi per la realizzazione dell'effetto parodico mettendone in evidenza le tecniche di realizzazione e le modalità più specificamente fumettistiche.

\section{1. L'italiano topolantico: tratti fonetici, morfologici e sintattici}

La riproduzione deformante dell' italiano antico da parte degli autori disneyani interessa diversi piani. A livello fonetico gli autori mostrano una predilezione per le forme non dittongate (es.: loco, core, infocate ecc., v. fig. 4), effettivamente presenti nella lingua della poesia fino al secolo scorso quali tracce del vocalismo siciliano antico. L'esistenza di forme simili nella lingua poetica si spiega ricordando che parole senza dittongo come còri, focu, nòvu, presenti nella lirica dei poeti siciliani delle origini (in cui le basi latine con $\breve{\mathrm{O}}$ e $\breve{\mathrm{E}}$ toniche non producevano dittongo), sono passate con alcuni aggiustamenti agli stilnovisti attraverso la mediazione della scuola siculo-toscana fino ad arrivare a Petrarca, per poi soppiantare nella tradizione poetica italiana le normali forme dittongate (cfr. Patota ${ }^{2} 2007$ : 6I-62). Gli sceneggiatori disneyani mostrano inoltre una predilezione per le forme piane di origine latina (provenienti da basi uscenti in -ATEM e-UTEM) come viltade, pietade, onestade rispetto alle parole tronche dell'italiano moderno (fig. 4). ${ }^{14}$ Non è sempre applicata invece in maniera sistematica la sonorizzazione dell'occlusiva dentale sorda intervocalica $-\mathrm{t}$ - che pure in italiano antico ha prodotto dalle basi latine in -ATEM / -UTEM la sillaba finale -de (> bontade, > cittade). Nella trasposizione disneyana invece gli esiti con consonante sorda e sonora si alternano in maniera casuale: "ma feci per viltate il gran rifiuto"; "senza pietade alcuna né rimorso"; "qui si parrà la mia nobilitate" ecc.

I4. In conseguenza di un'apocope aplologica, cioè della cancellazione della sillaba finale di parola quando questa era seguita da una preposizione di suono simile o identico nel concreto degli scambi comunicativi (ad es. in cittade de X, virtude de Y), le parole piane come cittade o virtude si sono trasformate in parole accentate sull'ultima sillaba come città o virtù. Sull'apocope aplologica cfr. Patota ${ }^{2} 2007$ : 99-100; I05-107, e D'Achille ${ }^{2} 2004$ : 70-72. 


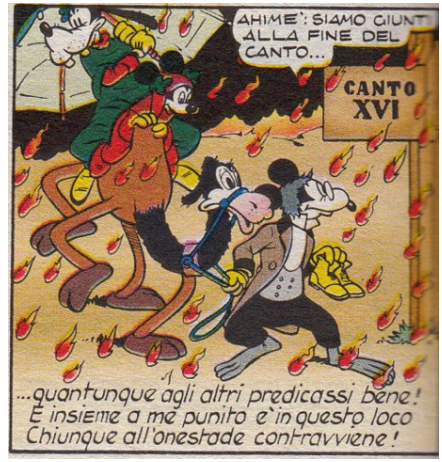

Fig. 4. Tra i tratti fonetici caratteristici dell'italiano pseudoantico di Topolino figurano forme non dittongate (loco) e parole piane di base latina (onestade).

La parodia disneyana riproduce inoltre alcuni casi di apocope sillabica (caduta di una sillaba in fine di parola) come pie' (< piede), fe' (< fece), ver' (< verso), e si esibisce in numerose apocopi vocaliche in linea con la tradizione letteraria italiana soprattutto in versi: pensier, passegger, legger insiem ecc.

La maggioranza dei tratti peculiari dell'italiano topolantìco riguarda la morfologia, piano linguistico sul quale più sembra sbizzarrirsi la fantasia deformatrice degli autori disneyani. A parte la sopravvivenza del plurale neutro di derivazione latina in -a in peccata ("Io chiesi ad un di lor: 'Quali peccata / Devi scontare, immerso in questo vasto, / Pantano freddo come una cassata?") che si ritrova anche nell'ipotesto dantesco ("e quel conoscitor de le peccata", If. $\mathrm{V}, 9$ ), il tratto morfologico distintivo della parodia disneyana è l'uso reiterato e caratterizzante dell'articolo definito maschile lo: lo solingo piano, lo fanale, lo Greco, lo buon maestro, lo peccator, lo loro invito ecc. Effettivamente l'italiano delle origini conosceva per l'articolo definito maschile singolare solo la forma lo come esito del latino ILLLŬM (con aferesi della sillaba iniziale $\breve{I L}$, caduta della M finale e passaggio della $\breve{U}$ a o), mentre la forma il si sarebbe prodotta successivamente. ${ }^{\text {I5 }}$ Nell'italiano del Trecento quindi, cui la parodia disneyana fa il verso, esistevano già le due forme moderne il e $l o$, ma il loro uso non corrispondeva a quello odierno. La selezione era determinata dalla finale della parola che precedeva l'articolo, a differenza di quanto accade nell'italiano moderno, in cui la distribuzione dipende dall'iniziale della parola che segue l'articolo. In particolare, secondo la cosiddetta "norma Gröber", si aveva il dopo parola terminante per vocale e lo a inizio frase e dopo parola terminante per consonante, un uso i cui residui persistono nelle espressioni

I5. Sulla sua origine esistono spiegazioni diverse: alcuni studiosi ipotizzano sempre a partire da ILLUM un'apocope (IL(LUM) $>e l>i l$ ), altri invece l'apocope di $l o$ a $l$ dopo una parola uscente per vocale nel parlato come fenomeno di "allegro", e quindi lo sviluppo di una vocale d'appoggio (cfr. D’Achille ${ }^{2}$ 2004: 82 e, per la seconda ipotesi, Patota ${ }^{2}$ 2007: I3I-I35). 
avverbiali perlopiù e perlomeno. Martina riprende il polimorfismo dantesco alternando le forme il e lo, ma, probabilmente ignorando la norma Gröber, la sua selezione è piuttosto casuale o modellata intuitivamente sull'ipotesto. Ritroviamo quindi nella parodia, proprio come in Dante ("Lo buon maestro "Acciò che non si paia / che tu ci sia', mi disse", If. XXI, 58-59; "Lo buon maestro disse: "Figlio, or vedi", If. VII, II5), l'articolo lo nel sintagma "lo buon maestro" ("Lo buon maestro in mezzo al parapiglia, / non fé come la torre che non crolla", oppure "Lo buon maestro allor mi disse: 'Vieni!"; v. anche fig. 5), ma mentre in Dante la presenza di lo si spiega con la posizione a inizio verso, lo sceneggiatore disneyano riproduce una sorta di sintagma fisso (lo buon maestro), tanto che altrove nella parodia l'articolo a inizio verso, in mancanza di un esplicito modello dantesco, non è più lo ma il ("Il verso mio di continuar rifiuta", oppure "Il pentimento nato nel suo cuore" ecc.). Altrettanto casuale appare il polimorfismo disneyano tra lo ciel e il ciel ("Quivi lo ciel pareva più sereno" vs. "Messer pollastro, è il ciel che v'ha mandato"), a dimostrazione di come si tratti appunto di una deformazione parodica dell'italiano trecentesco che ne echeggia intuitivamente le caratteristiche senza pretese di rigore sistematico.

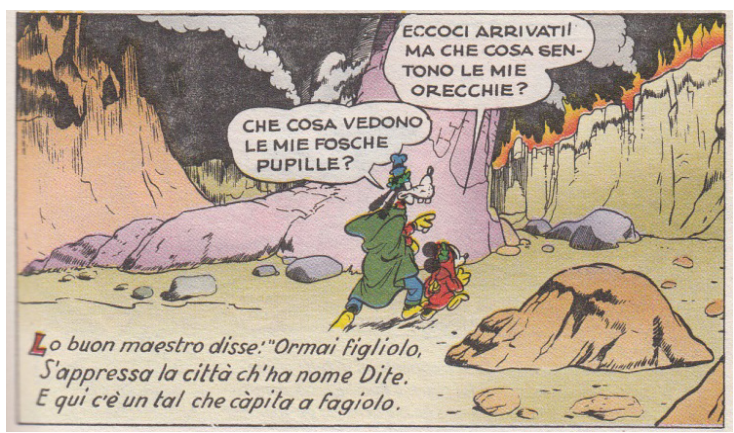

Fig. 5. La parodia riprende l'uso antico dell'articolo definito $l o$, sia pure con qualche incongruenza rispetto all'italiano del Trecento.

Nel segno del polimorfismo si collocano anche le scelte dei pronomi personali di terza persona nel testo della parodia, con una netta preferenza per egli e diverse attestazioni anche della forma più rara ei ("Ei salutommi con il più sincero / 'Caramba' pronunciato in messicano"; "Ei fronteggiò la casa dei meschini, / poi con il petto e con le gote enfiate, / gridava sogghignando: 'Poverini!'”). Un'analoga ricchezza di forme si ritrova nei dimostrativi del fumetto, che accolgono non soltanto il sistema tripartito tradizionale questo / codesto / quello (che resiste tra l'altro nel fumetto disneyano in generale, indipendentemente dall'intento parodico, cfr. Pietrini 2009: 340-352), ma persino

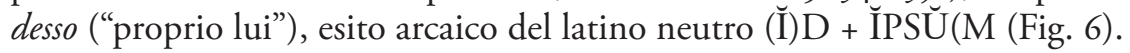




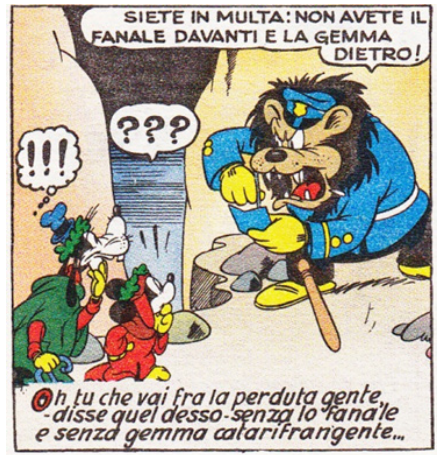

Fig. 6. Sopravvive nella parodia disneyana persino il dimostrativo arcaico desso.

Un cenno a parte meritano le forme verbali della parodia disneyana. L'insistenza sul passato remoto si realizza nel pieno rispetto delle forme di terza persona plurale dell'italiano delle origini. Troviamo così nel fumetto le forme arcaiche in -iro ("Nuovi prodigi all'occhio ci appariro!"), esito della desinenza plurale latina -(VE)R $\breve{(N T)}$ con accento ritratto secondo la pronuncia del latino colloquiale, che ha generato la sincope della sillaba VE e la caduta di NT (cfr. Patota 2007: 159), mentre mancano quelle analoghe in -aro ("e odiarono la gente troppo gaia"; "sovra di noi calarono ad ondate" ecc.). Per quanto riguarda l'imperfetto indicativo la parodia preferisce le forme del fiorentino trecentesco in -ea / -ia senza labiodentale: finia, avea, scomparia, cadea... ("Tu dèi saper ch'io fui Conte Ugolino / ed arbitravo a Pisa una partita / ch'avea in palio il titol di campione..."). ${ }^{16}$

Quanto alla riproduzione della sintassi dell'italiano antico, spicca nella parodia disneyana la tendenza all'espressione del pronome personale soggetto, non obbligatoria nel latino classico né nell'italiano moderno, a differenza di molte lingue e dialetti romanzi: "Nei testi toscani antichi il pronome soggetto tende infatti all'obbligatorietà, propria del fiorentino moderno" (D'Achille 22004: 106). Anche nella parodia disneyana il pronome personale soggetto è spesso espresso non soltanto nelle subordinate ("Il terzo che tu vedi è un imbroglione"; "ed ora, giunti al doloroso ostello, / come tu vedi, sono condannati", ecc.), ${ }^{17} \mathrm{ma}$ anche in numerose frasi principali di tipo dichiarativo: "io rimirai la livida fungaia"; "noi ci affacciammo tosto sovra il tetro / girone ardente di color sanguigno"; "ma questo che tu vedi mi consola, / e noi faremo come l'aviatore, / che sugli altri come aquila vola!" e molti altri esempi.

I6. Sull'evoluzione fonetico-morfologica dall'imperfetto latino alle forme dell'italiano arcaico v. almeno D'Achille 2004: 94-95.

17. Per un'esposizione più dettagliata delle complesse regole per l'espressione del soggetto in frase subordinata nell'italiano antico cfr. di Salvi/Renzi 20IO: 43-47. 
Il tratto sintattico più marcato cui lo sceneggiatore disneyano si affida per riprodurre l'italiano antico è l'enclisi dei pronomi personali atoni (cioè l'appoggiarsi dei pronomi atoni al verbo che li precede come in dissegli e non, come nel contemporaneo gli disse, al verbo che li segue). L'enclisi dei pronomi atoni, descritta per il francese e l'italiano antichi dalla cosiddetta "legge ToblerMussafia”, era obbligatoria ancora per tutto il Trecento se il verbo coniugato occupava la prima posizione nella frase, dopo le congiunzioni $e$ e $m a$, e quando il verbo era preceduto da una subordinata circostanziale (cfr. Salvi / Renzi 20I0: 54-59; 432-436), ma era facoltativa in tutti gli altri casi, e come enclisi libera è sopravvissuta nella tradizione letteraria italiana ancora fino a tutto l'Ottocento. Nella parodia dell' Inferno l'enclisi è applicata sistematicamente: "Poi tosto il corpo suo divenne trino, / e sovra ognun cambiava il becco e il muso / così che tramutossi in Paperino!"; "La volpe dileguossi negli oscuri / antri, e i ragazzi furono sereni, / sotto l'usbergo del sentirsi puri!"; "Ma mentre salivan sulla sua nave, / gridocci: 'Chi son questi sbafatori / che vivi stanno fra le genti ignave?"'; "Poi quando il gonfiamento fu compito, udissi una terribile esplosione!" e la serie degli esempi potrebbe continuare ancora a lungo (v. anche fig. 7).

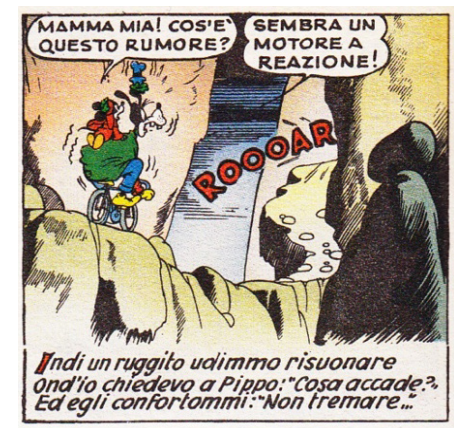

Fig. 7. La lingua della parodia disneyana applica costantemente l'enclisi dei pronomi personali atoni, fenomeno sintattico tipico dell'italiano antico.

\section{2. Il lessico topolantico}

Anche sul piano lessicale "L'Inferno di Topolino" riprende l'italiano trecentesco basandosi sulla duplice strategia linguistica di riprodurre in senso arcaizzante tanto il lessico funzionale quanto quello referenziale dell'italiano del Trecento. Da un lato la parodia abbonda di latinismi e termini aulici e letterari quali favella, desio, avello, usbergo, appropinquare, augello, periglioso, lasso, manducare, solingo ecc. Ma più che il ricorso a un lessico referenziale di varia ispirazione dantesca, ${ }^{18}$ fondamentale per la percezione della letterarietà

18. Tra l'altro la profusione di termini aulici e arcaizzanti non si limita al filone delle Grandi Parodie inaugurato con "L'Inferno di Topolino", ma caratterizza tutto il linguaggio del 
ludica dell'ipertesto disneyano e per la riuscita della deformazione dell'ipotesto dantesco è l'uso di una vasta gamma di parti invariabili del discorso (preposizioni, avverbi, congiunzioni) di sapore arcaico. Ė quindi su questo aspetto che ci soffermeremo nelle brevi osservazioni che seguono.

La riproduzione dissacrante della lettera della cantica dantesca passa attraverso la sostituzione sistematica del lessico funzionale dell'italiano contemporaneo con quello dell'italiano trecentesco selezionando accuratamente non le forme comuni, ma quelle antiquate o addirittura in disuso. Figurano quindi nel testo narrativo (didascalie) della parodia alcune tra le congiunzioni subordinanti causali e finali più comuni dell'italiano antico quali imperocché ("Nel quale il passegger vede le stelle / imperocchè viene compresso al punto / che dalle fauci gli escon le budelle!"), acciocchè ("laggiù poi pregheremo Satanasso / acciocchè sia con noi tanto cortese / da farci uscir dal doloroso passo") o la congiunzione relativa con valore causale/finale onde: "Indi un ruggito udimmo risuonare / ond'io chiedevo a Pippo: 'Cosa accade?' / Ed egli confortommi: 'Non tremare..."' (v. fig. 7). Per quanto riguarda le subordinate temporali, la parodia disneyana ricorre a due introduttori tipici dell'italiano antico: tosto (di solito seguito dalla congiunzione $c h e$ ) per indicare la precedenza immediata dell'evento espresso dalla subordinata: "Tosto che dalla nave fummo scesi, / ponemmo il piede in una stretta gola"; "Tosto che il fumo intorno fu svanito, / vedemmo il lupo nudo e secco secco", 19 e poscia (che) per esprimere l'anteriorità: "Poscia che l'ebbe ben considerato, / disse contento: 'Siate il benvenuto". La parodia disneyana non rinuncia nemmeno al costrutto concessivo introdotto da avvegna che (scritto univerbato): "e avvegnaché la fame sempre gli urga / il ventre vuoto come una bisaccia, / resta digiuno e gli si dà la purga!". Da segnalare anche il ricorso nella parodia ad alcuni avverbi locativi non deittici (la cui identificazione era affidata esclusivamente al rinvio al contesto linguistico) tipici dell'italiano antico quali quivi ("quivi lo ciel pareva più sereno"; "quivi giacevan le perdute genti"; cfr. fig. 8) e indi ("indi una mano fra la sua mi prese"; v. anche fig. 7), oltre al deittico spaziale quinci ("quinci lasciammo l'infocate prode / per riposarci dopo la partita / da cui uscimmo con cotanta lode"; "quinci volgemmo le veloci rote / e in un baleno fummo colaggiù / ove s'udìan le dolenti note"), anch'esso tipico dell'italiano antico, ma assente nell'italiano moderno (sugli avverbi di luogo e la deissi spaziale nell'italiano antico cfr. Salvi / Renzi 20I6: I248-I263). All'elenco possiamo aggiungere altre preposizioni e avverbi rari e arcaici come colaggiù, giuso ("così, scendendo giuso nel profondo, / dal primo cerchio Pippo il piede mosse, / e

fumetto Disney made in Italy costituendo una delle principali tecniche costruttive della sua comicità. Cfr. in proposito Pietrini 2009: 97-II5.

19. Sull'espressione della precedenza immediata in italiano antico cfr. Salvi / Renzi 20ı6: 961-962. 
tosto m'introdusse nel secondo") e anche quaggiuso ("nel chiudere il portello del forziere / è questo degli avari il triste dramma: / quaggiuso son ridotti al miserere") ecc.

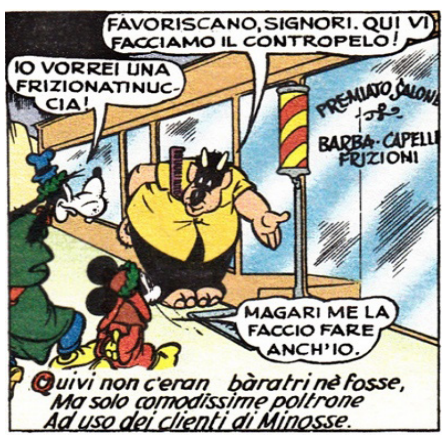

Fig. 8: Fondamentale per la ricreazione deformante dell'italiano dantesco è l'uso di un lessico funzionale arcaizzante.

\section{Lo STILE TOPOLDANTESCO}

La parodia disneyana dell' Inferno non si limita al tentativo di imitare il linguaggio poetico dell'italiano trecentesco. Alla caricatura di alcuni tratti fonetici, morfologici, sintattici e lessicali tipici dell'italiano del Medioevo gli autori aggiungono infatti una ricostruzione ludica dello stile dantesco basata essenzialmente su due espedienti: da un lato la ripresa quasi letterale di alcuni versi di Dante con leggere modifiche (volta ad attivare il riconoscimento della lettera dell'ipotesto nel travestimento ipertestuale), dall'altro la ripetizione quasi ossessiva di alcune citazioni emblematiche con effetto comico.

A essere citati a mo' di ritornello in un incessante gioco di repetitio e variatio sono alcuni versi e sintagmi danteschi che, decontestualizzati, assumono il ruolo di veri e propri topoi "infernali". Tra i più sfruttati figura l'espressione (ri) veder le stelle, che scandisce la narrazione parodica già dalla prima vignetta (in cui la citazione è funzionale a stabilire la relazione ipertestuale del fumetto con il suo ipotesto e il legame ludico tra testo e immagine, v. $\$$ 3.I.) e altrove nella cornice dialogica ("la tua carezza fa veder le stelle!"), passando per le fasi intermedie della parodia: "per poco Pippo non lasciò la pelle / che fra i due litiganti il terzo gode, / e lui soltanto può veder le stelle!", fino alla conclusione "il ciel per te s'accenda di fiammelle / splendenti a rischiararti ancor la via, / sì che tu possa riveder le stelle!". Anche la citazione del celeberrimo "nel mezzo del cammin di nostra vita" trova posto nella parodia disneyana tanto nella cornice iniziale: "o Beatrice, ancor non è finita quella commedia ch'era incominciata nel mezzo del cammin di nostra vita!" che nel corso della narrazione: 
"e quella fu la tregua più gradita / da quando cominciammo il grande viaggio / nel mezzo del cammin di nostra vita". Non poteva mancare la celeberrima "selva" dantesca, tanto "oscura" ("Come nel primo canto v'ebbi a dire, in una selva oscura mi trovai / che nel pensier mi fa rabbrividire", o anche "viaggiando in questo modo comodissimo / noi ci addentrammo nella selva oscura / per un sentier tortuoso e ripidissimo") quanto "ingrata" ("e chi è punito in questa selva ingrata?"; "ci ridurranno in miseri rottami / che nella selva ingrata nuovamente, / ritorneranno ad esser tronchi e rami!"; "usciti fuori della selva ingrata / giugnemmo ai bordi d'un'immensa piana / in cui cadea un'ardente nevicata..."), o ancora il verso "lasciate ogni speranza, voi ch'intrate" (If. III, 9), ripreso in: "d'esser sani e salvi non crediate! / Quando a una porta s'avvicina il lupo / lasciate ogni speranza, o voi ch'entrate!". Il testo della parodia disneyana non echeggia solo interi versi dell'Inferno dantesco, ma anche brevi sintagmi. Si citino qui a titolo di esempio "perduta gente" ("quivi giacevan le perdute genti / supine dentro a scoperchiati avelli / da fiamme esterne resi incandescenti") o "dolente regno" ("e tutto rimbombò il dolente regno").

Nel gioco delle citazioni gli autori mescolano il travestimento parodico dell'ipotesto con altri elementi della tradizione lirica italiana danteschi e non. Ecco quindi decontestualizzazioni di versi di altre cantiche della Commedia come l"'era già l'ora che volge il disio" (da Pg. VIII, I) in: "era già l'ora che volge'l disio / a bimbi di trovarsi insieme ai padri / uniti attorno a un desco dolce e pio...", di altri componimenti dello stesso Dante (Io dissi allora: 'Maestro, non tremare! / Non vedi che la bestia che t'angoscia / tanto gentile e tanto onesta pare?!'), o addirittura di altri autori della tradizione poetica italiana ('io sono quei che colse laude e allori, / - risposi a lui - cantando in rima pura / le donne, i cavalier, l'armi, gli amori", citazione demistificata tra l'altro dalle battute nelle nuvolette, v. fig. 9).

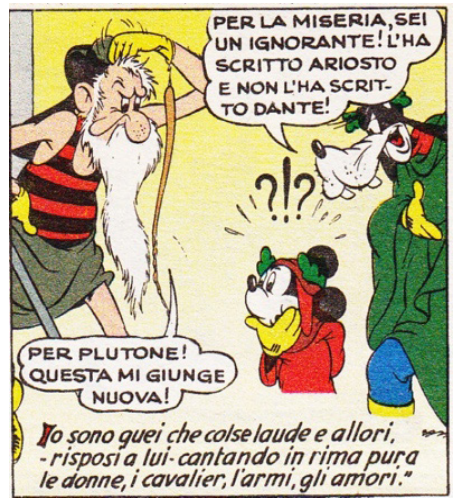

Fig. 9.: Lo stile della tradizione poetica italiana viene riprodotto giocando con citazioni modificate o falsamente attribuite. 
Altre volte invece il passo celebre non viene affatto citato, ma piuttosto travisato in maniera allusiva se non addirittura stravolto nella lettera, richiedendo così l'intervento del lettore a riconoscere il travolgimento parodico dell'ipotesto. Il gioco può funzionare solo con versi particolarmente noti, come quello che chiude il canto V "e caddi come corpo morto cade", che nella parodia disneyana diventa "ma cadde come l'uom cui sonno piglia!".

\section{LA PARODIA TRA TESTO E DISEgNO}

Finora abbiamo analizzato "L'Inferno di Topolino" come se si trattasse di un'opera di solo testo, illustrando gli strumenti linguistici con i quali viene riprodotto l'italiano poetico del Trecento e i mezzi per citare - deformandolo - lo stile dantesco. Eppure una mera imitazione, sia pur caricata, non basterebbe a garantire l'effetto comico, e comunque non renderebbe giustizia all'operazione di Martina e Bioletto, molto più complessa. È infatti proprio lo specifico fumettistico a rendere la parodia disneyana qualcosa di unico. Il fumetto, grazie alla pluralità di luoghi deputati all'elemento verbale (didascalia, nuvoletta, ideofoni, testo disegnato, cfr. $\$$ 3.I.) e al rapporto di costruzione reciproca del senso tra testo e immagine, può moltiplicare i piani narrativi e basare l'effetto parodico proprio sullo scarto tra i livelli. Lo stravolgimento in senso ludico della lettera dell'ipotesto dantesco attraverso aulicismi e latinismi, morfologia e sintassi pseudo-trecentesche, citazioni martellanti e deformate e tutti gli altri elementi illustrati nei paragrafi precedenti non occupa che una parte della parodia, inserito in una sorta di didascalia a piè di vignetta che richiama in maniera esplicita il fumetto italiano delle origini. ${ }^{20}$ L'intera parodia disneyana dal punto di vista linguistico non si basa quindi (sol)tanto sulla deformazione caricaturale dell'italiano antico e dello stile di Dante, ma piuttosto sul corto circuito ludico tra il linguaggio aulico così (ri)costruito e il registro colloquiale e familiare delle nuvolette dialogiche, in un gioco di raddoppiamento che sortisce l'effetto di abbassare il tono smitizzando l'ipotesto e generando l'effetto comico. Il terzo livello è rappresentato dall'immagine, che contribuisce a dissacrare il modello attraverso la rappresentazione visiva di situazioni assolutamente prosastiche in forte scarto con il linguaggio aulico-letterario delle didascalie che vi fanno quasi da sottotitolo. Il raffronto diretto tra le vignette (fig. Io), le nuvolette e le didascalie (tab. I) dell'episodio iniziale dell'incontro tra Topolino-Dante e Pippo-Virgilio basti a illustrare questo procedimento che si snoda per l'intero fumetto.

20. Ricordiamo che le nuvolette furono bandite dai primi fumetti italiani per trasformarsi in castigate didascalie formate da distici di ottonari a rima baciata o alternata (es.: Il signor Bonaventura di Tofano, Bilbolbul e gli altri personaggi di Rubino o Mussino), probabilmente ritenute più colte e meno rivoluzionarie delle nuvolette. 

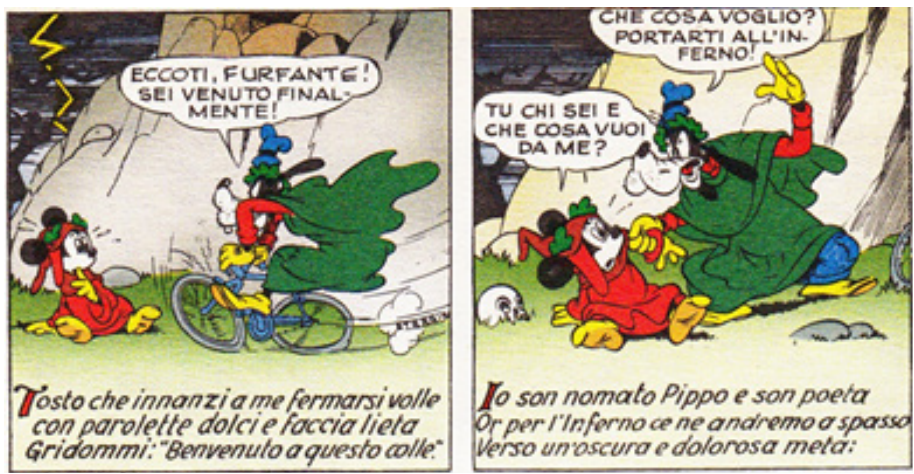

Fig. Io. I disegni raffiguranti l'incontro tra Topolino-Dante e Pippo-Virgilio sviluppano tutto il loro potenziale ludico solo nello scarto con il registro pseudo-letterario delle didascalie.

\begin{tabular}{|l|l|}
\hline $\begin{array}{l}\text { Didascalia } \\
\text { Italiano aulico pseudo-trecentesco }\end{array}$ & $\begin{array}{l}\text { Nuvoletta } \\
\text { Italiano colloquiale }\end{array}$ \\
\hline $\begin{array}{l}\text { con parolette dolci e faccia lieta gri- } \\
\text { dommi: "Benvenuto [...]" }\end{array}$ & eccoti furfante! sei venuto finalmente! \\
\hline or per l'inferno ce ne andremo a spasso & che cosa voglio? portarti all'inferno! \\
\hline
\end{tabular}

Tab. I: Lo stravolgimento parodico nasce dall'abbassamento di tono che risulta dallo scarto tra il registro delle didascalie e quello del testo nelle nuvolette dialogiche.

\section{OsSERVAZIONI CONCLUSIVE}

L'analisi condotta ha dimostrato il ruolo fondamentale dell'elemento verbale per la riuscita della trasposizione parodica dell'Inferno dantesco in un fumetto come "L'Inferno di Topolino". Dal punto di vista linguistico il necessario stravolgimento deformante della lettera dell'ipotesto si realizza attraverso la ricreazione caricata di un italiano poetico pseudo-trecentesco per la cui realizzazione lo sceneggiatore utilizza sapientemente alcuni saliency markers dell' $\mathrm{i}-$ taliano antico (tratti fonetici, morfologia verbale e pronominale, enclisi dei pronomi atoni, particolare ordine delle parole ecc.). Alla presunta letterarietà dell'ipertesto parodico contribuisce in maniera determinante un lessico arcaizzante non solo dal punto di vista referenziale, ma anche per quanto riguarda le parti invariabili del discorso, in cui congiunzioni, preposizioni e avverbi moderni vengono sistematicamente sostituiti con equivalenti antiquati o addirittura scomparsi. La ripetizione martellante di alcune citazioni emblematiche del testo della Commedia e la loro deformazione ludica contribuisce a ricreare uno stile pseudo-dantesco grazie all'invenzione di una sorta di "ritornelli" poetici che scandiscono la parodia dalla cornice all'epilogo. Eppure l'imitazione dell'italiano del Trecento non è l'unico ingrediente della parodia a fumetti. 
Fondamentale per la riuscita del gesto parodico è soprattutto lo scarto diafasico tra registri contrapposti, reso possibile dalla molteplicità degli spazi che il genere testuale del fumetto riserva all'elemento verbale (soprattutto didascalia narrativa e nuvoletta dialogica), raddoppiato dalla raffigurazione disegnata di situazioni altamente prosastiche, anch'esse in netto contrasto con il registro aulico del testo a piè vignetta. È quindi nello specifico del fumetto in quanto narrazione sequenziale di tipo dialogico basata sull'interazione paritaria di un elemento verbale con un elemento disegnato che il gesto parodico trova il suo compimento. 


\section{RIFERIMENTI BIBLIOGRAFICI CITATI}

AA.VV., s.d., "Parodie Disney", PaperPedia Wiki, http://it.paperpedia.wikia.com/wiki/ Parodie_Disney (ultima consultazione 22-6-2018).

Alighieri, D., 1967, La Divina Commedia. Inferno, a cura di G. Giacalone, Roma, Signorelli.Ov.

Becattini, A./Boschi, L./Gori, L./Sani, A., 20I2, I Disney italiani, Roma, NPE.

Brambilla, A., 2013, "Le origini de 'L'Inferno di Topolino"? In un diario scolastico", Fumettologica, www.fumettologica.it (ultima consultazione 5-7-2018).

Buttitta, A., 2015, "750 anni dalla nascita di Dante. Dieci rivisitazioni pop della 'Divina Commedia': dalle serie tv ai fumetti”, Huffington Post, https:/www.huffingtonpost.it/2015/02/03/divina-commedia-rivisitazioni-pop_n_6603334.html (ultima consultazione 20-6-2018).

Chiamenti, M., s.d., "Dante e il cinema”, http://www.nuovorinascimento.org/n-rinasc/saggi/pdf/chiament/dantecinema.pdf (ultima consultazione 20-6-20I8).

Cotugno, A./Gargano, T., 2016, Dante pop. Romanzi, parodie, brand, canzoni, Bari, Progredit.

D’Achille, P., ${ }^{2} 2004$, Breve grammatica storica dell'italiano, Roma, Carocci.

Detti, E., 1984, Il fumetto tra cultura e scuola, Firenze, La Nuova Italia.

Frezza, G., 1987, La scrittura malinconica: sceneggiatura e serialità nel fumetto italiano, Firenze, La Nuova Italia, 45-52.

—, I989, "Il fumetto", Letteratura Italiana. Storia e geografia. III: L'età contemporanea, Torino, Einaudi.

Garavelli, B., s.d., "Dante e la Commedia nel cinema”, www.treccani.it/scuola/tesine/ divina_commedia_e_iconografia/ıo.html (ultima consultazione 3-I2-20I5).

Genette, G., 1982/1997, Palinsesti. La letteratura al secondo grado, Milano, Einaudi.

Groensteen, T., 20I0, Parodies - La Bande Dessinée au second degré, Paris, Skira Flammarion.

Householder, F.J., I944, "Parodia", Classical Philology XXXIX, I, I-9.

Idt, G. (I972-73): "La parodie: rhétorique ou lecture?", Le Discours et le sujet 3, I28-I73.

Inducks, L'Archivio mondiale dei fumetti Disney, inducks.org (ultima consultazione 30-7-20I8).

Napolitano, A., 20I6, "Quando l'Inferno di Dante diventa pop: film, romanzi, fumetti e videogiochi”, http://www.roarmagazine.it/divina-commedia-pop/ (ultima consultazione 20-6-2018).

NDMo: Nuovo De Mauro online, ospitato sul sito Internazionale, https://dizionario. internazionale.it (ultima consultazione 3I-7-20I8).

Pacifico, R., 20I5, "Ritratto dell'artista da giovane settecentocinquantenne", Quaderni d'altri tempi, 57, www.quadernidaltritempi.eu (ultima consultazione 30-7-20I8).

Pallavicini, R., 20I6, "Indagini infernali. Gli inferni di Martin Mystère, Lazarus Redd e Nathan Never", http://www.arabeschi.it/-inchieste-seriali-misteri-e-universiparalleli-3I-indagini-infernali-gli-inferni-di-martin-mystre-lazarus-ledd-enathanneverdi-renato-pallavicinitre-sto/ (ultima consultazione 22-6-20I8).

Patota, G., ${ }^{2} 2007$, Nuovi lineamenti di grammatica storica dell'italiano, Bologna, Il Mulino.

Pietrini, D., 2009, Parola di papero. Storia e tecniche della lingua dei fumetti Disney, Firenze, Cesati.

—, 20I4, "Les marqueurs discursifs dans l'oralité fictive de la bande dessinée", in: Weidenbusch, W. (a c. di), Diskursmarker, Konnektoren, Modalwörter, Tübingen, 
Narr: 85-106.

Quartu, M./Rossi, E., 20I2, Dizionario dei modi di dire della lingua italiana, Milano, Hoepli.

Quintilianus, M.F., I822, De Institutione oratoria (a cura di Jean Joseph F. Dessault, Georg Ludwig Spalding).

Sabatini, F./Coletti, V., 2005, (a c. di), Il Sabatini Coletti 2006. Dizionario della lingua italiana, Milano, Rizzoli-Larousse.

Salvi, G./Renzi, L., 20Io, (a c. di), Grammatica dell'italiano antico, Vol. I e II, Bologna, Il Mulino.

Sangsue, D., 2006, La parodia (a cura di Fabio Vassarri), Roma, Armando (orig. francese 1994: La parodie, Paris, Hachette).

TLFi: Le Trésor de la Langue Française informatisé, http://atilf.atilf.fr/tlf.htm (ultima consultazione 26-6-20I8).

TREC : Vocabolario online Treccani, http:/www.treccani.it/vocabolario/ (ultima consultazione 30-7-2018).

\section{FUMETTI CITATI}

Burattini, M./Longo, F., 20I6, La porta dell'Inferno, Speciale Dampyr I2, Milano, Sergio Bonelli.

Burattini, M./Sommacal, G., I992, Un'avventura infernale, Cattivik 32, Milano, Macchia Nera.

—, I993, Cattivik in Purgatorio, Cattivik 40, Milano, Macchia Nera.

-, I994, Cattivik in Paradiso, Cattivik 54, Milano, Macchia Nera.

Capone, A./Bartolini,F./Bocci, A., 2000, Discesa all'Inferno, Lazarus Ledd 89, Bosco (PG), Star Comics.

Dossi, S., 20II, Geppo - Inferno 2000, numero da collezione autoprodotto dall'autore (ma già 1984, Geppo II6, Milano, Editoriale Metro).

Jacovitti, B., I947, La rovina in commedia. Grottesco satirico e dantesco, Belzebù, Roma, GIT.

Marconi, M./ Chierchini, G., I987, L’Inferno di Paperino, Topolino I654.

Martina, G./Bioletto, A., I949-I950, L'Inferno di Topolino, Topolino 7-I2.

Martina, G./Carpi, G.B., I980, Paolino Pocatesta e la bella Franceschina, Topolino I26I.

Rota, M., 1986, I grandi di Paperino mese: Paperante Alighieri, Paperino mese 70.

Santarelli, S./Torti, R., I994, Diavoli dell'Inferno!, Martin Mystère I53, Milano, Sergio Bonelli.

Toninelli, M., 2015, Dante. La Commedia a fumetti, Bientina (PI), Shockdom.

Vigna, B./Bastianoni, D., I992, Inferno, Nathan Never Io, Milano, Sergio Bonelli. 